\title{
Contact Lens Classification by Using Segmented Lens Boundary Features
}

\author{
Nur Ariffin Mohd Zin ${ }^{1}$, Hishammuddin Asmuni ${ }^{2}$, Haza Nuzly Abdul Hamed ${ }^{3}$, Razib M. Othman ${ }^{4}$, \\ Shahreen Kasim ${ }^{5}$, Rohayanti Hassan ${ }^{6}$, Zalmiyah Zakaria ${ }^{7}$, Rosfuzah Roslan $^{8}$ \\ ${ }^{1,5,8}$ Soft Computing and Data Mining Centre, Faculty of Computer Science and Information Technology, \\ Universiti Tun Hussein Onn Malaysia \\ ${ }^{2,3,4,6,7}$ Laboratory of Computational Intelligence and Image Processing, Faculty of Computing, \\ Universiti Teknologi Malaysia
}

\begin{tabular}{l} 
Article Info \\
\hline Article history: \\
Received May 2, 2018 \\
Revised Jun 9, 2018 \\
Accepted Jun 21, 2018 \\
\hline Keywords: \\
Contact lens classification \\
Local descriptor \\
Support Vector Machines
\end{tabular}

Article Info

Article history:

Received May 2, 2018

Revised Jun 9, 2018

Accepted Jun 21, 2018

\author{
Local descriptor
}

\begin{abstract}
A Recent studies have shown that the wearing of soft lens may lead to performance degradation with the increase of false reject rate. However, detecting the presence of soft lens is a non-trivial task as its texture that almost indiscernible. In this work, we proposed a classification method to identify the existence of soft lens in iris image. Our proposed method starts with segmenting the lens boundary on top of the sclera region. Then, the segmented boundary is used as features and extracted by local descriptors. These features are then trained and classified using Support Vector Machines. This method was tested on Notre Dame Cosmetic Contact Lens 2013 database. Experiment showed that the proposed method performed better than state of the art methods
\end{abstract}

\section{Corresponding Author:}

Nur Ariffin Mohd Zin,

Soft Computing and Data Mining Centre,

Faculty of Computer Science and Information Technology,

Universiti Tun Hussein Onn Malaysia.

Email: ariffin@uthm.edu.my

\section{INTRODUCTION}

Since the foundation of iris recognition devised by Daugman [1], it is then categorized under the most reliable biometrics exist [2-4]. This is supported by Wang and Han [5] whose stated 3 traits of iris biometrics; Firstly, iris is an internal body which protected, secondly, it cannot easily being altered without damaging its vision and lastly the dilation due to illumination response makes it hard to imitate. Although being considered as reliable, there are still open issues in iris recognition. Among them, one that could be how the recognition reacts towards the wearing of contact lens, whether it is soft or cosmetic lens. This issue has been thoroughly studied in [6] and [7], that apart from cosmetic lens, soft lens can leave significant degradation during verification. From their findings, the false reject rate for matching the same subject, by considering with soft lens as probe image and without soft lens as gallery image has resulted in 5.66\% FRR. As a comparison, matching without lens and soft lens for both gallery and sensor images only resulted in $1.17 \%$ and $1.67 \%$ respectively.

To handle the emerging of 125 million contact lens wearers [8], it is crucial that one recognition system should have an early stage mechanism to detect the presence of contact lens [9], and what type the lens is. It is also statistically proven that a recognition system performance degrades while recognizing contact lens subjects [10], [11]. Unlike cosmetic lens, soft lens is worn to correct eye vision rather than for appearance purpose. Soft lens is usually colourless, while cosmetic lens may appear in wide variety of colours. Besides, soft lens is imperceptible unless been inspected carefully. Hence, detection of soft lens 
would be a non-trivial task that requires a mathematical and statistical approach to automating the task. The presence of soft lens can be noticed for one distinct feature, which is the lens boundary located on top of the sclera region. We may assume this boundary can be easily detected, however, it comprises of very thin line and can be easily confused by noise resulted from inconsistent illumination. In this work, we take the challenge to segment the lens boundary and use it as features, then, train it using Support Vector Machine, in order to classify between with or without soft lens. The novelty of our work is the fusion of extracted features produces from two prominent descriptors, Histogram of Gradient (HOG) and Scale Invariant Feature Transform (SIFT). The details of our work are represented in the following sections. In Section 2, any previous works regarding contact lens detection are discussed. In Section 3, our proposed method is described in details. The experimental results and discussions are reported in Section 4 and lastly, Section 5 draws the conclusion.

\section{RELATED WORKS}

During decades, cosmetic lens has gained a lot of attention in iris recognition community. A lot of research has been conducted extensively mainly under the subject of fake iris detection and iris spoofing. It was pioneered by Daugman [12] who managed to detect dot matrix cosmetic lens using Fourier transform. Then, Lee et al. [13] introduced the use of Purkinje image to detect fake iris. The research continues where He et al. [12] utilized gray-level co-occurrence matrix (GLCM) as feature descriptor and SVM as classifier. They reported $100 \%$ accuracy tested on self-database. Meanwhile, Wei et al. [15] proposed three methods for contact lens detection; iris edge sharpness, iris-textons and GLCM with SVM. Evaluation is done by using CASIA and BATH database with accuracy achieved above 76.8\%. Zhang et al. [16] used SIFT-weighted Local Binary Pattern with SVM as classifier. They achieved 99\% accuracy using self-database. Unlike cosmetic lens, soft lens has gained less attention in the community. This is due to the believe that soft lens wearing does not cause significant impact of degradation during wearing as supported in [17-19]. However, the awareness of the soft lens's wearing impact in [6], [20] has ignited more researches being conducted.

There are three approaches to soft lens detection, whether it is hardware, machine learning or image segmentation approach. Hardware approach requires the use of sophisticated camera. Such examples are from Kywe et al. [21] where they used a thermal camera to measure the decrement of temperature on the eye surface during the blinking of the eye. They observed that a certain degree of decrement indicates the wearing of soft lens. Another work by Lee et al. [22] claimed that Purkinje images between original and lens worn iris are difference. These images are captured using two collimated IR-LED cameras. Recent work by Hughes and Bowyer [23] detect the presence of lens by using stereo vision from two cameras. Soft lens wearing is detected if the captured image seen as curved surface rather than flat (without lens).

Meanwhile, machine learning requires features descriptor and classifier to perform. Doyle et al. [9] used a modified Local Binary Pattern (LBP) as features descriptor and experimented with 14 different classifiers. They achieved $96.5 \%$ of correct classification for cosmetic lens. However, only 50.25\% correct classification for soft lens. Kohli et al. in [24] experimented with four methods; iris edge sharpness, textural features based on co-occurrence matrix, gray level co-occurrence matrix and LBP with SVM. They reported that LBP with SVM has inferred the best result in overall. However, only 54.8\% CCR achieved for soft lens. Later, Yadav et al. [7] extend the work in [9] and [24] with additional database and revised algorithm. They achieved CCR above 45.35\%. Gragnaniello et al. in [25], [26] used sclera and iris region as features and applied Scale Invariant Descriptor as feature descriptor with SVM as classifier. They reported CCR above 76.29\%. Raghavendra et al. [27] proposed the use of Binarized Statistical Image Features (BSIF) with SVM. They achieved $62 \%$ for intra-sensor and 54\% accuracy for inter-sensor. Silva et al. [29] used Convolutional Neural Network. They resulted in $65 \%$ for intra-sensor and $42.25 \%$ for inter-sensor.

On the other hand, image segmentation approach uses edge detection technique to segment the thin lens boundary located on top of the sclera region. As to date, Erdogan and Ross [29] are the pioneer to implement this approach. They proposed a clustering based edge detection to segment the outer lens boundary which has achieved an accuracy of $70 \%$. From the literature, it can be summarized that the hardware approach relies on the physical characteristic of the eye. Machine learning approach instead involves the discrimination between two templates [30] while image segmentation deals with pixels manipulation.

\section{PROPOSED METHOD}

In this work, we proposed a fusion of image segmentation and machine learning approach to classify two class problems of validating the presence of with or without soft lens. These approaches are chosen as machine learning has the ability to extract and learn from training data to infer decision for a new data as well

Indonesian J Elec Eng \& Comp Sci, Vol. 11, No. 3, September 2018 : 1129 - 1135 
as image segmentation approach that robust to lens type and sensors. Careful inspection shows that the wearing of soft lens will leave an obscure or darker edge line on top of the sclera region as shown in Figure 1. It has been thoroughly studied in [25] that the sclera region define the best sample to prove the presence of soft lens. Therefore, our focus is to extract information on this region by locating the pixels of lens boundary and transform it into feature space by mean of a feature descriptor. These features are then to be trained by a statistical learning approach in order to be classified. The next sub-section presents the main three stages of the proposed method which are segmentation, features extraction and classification. The step by step processes of this method is depicted in Figure 2 .


Figure 1. Examples of soft lens boundary location as pointed by arrow

\subsection{Segmentation}

In the segmentation stage, the main objective is to locate the soft lens boundary which is deemed to reside on top of the sclera. It starts with segmentation of the left and right sclera region. We used the radius information provided with Notre Dame Cosmetic Contact Lens 2013 (NDCCL13) [31] to segment the iris boundary by the mean of Circular Hough Transform. Then, we extend the iris radius with additional 30 pixels to include the sclera region concentrically. The right-side region is segmented between the upper value of 150 to lower value of 210 degree rotation and 30 to -30 degree rotation respectively at the left-side. Therefore, the segmentation process will produce two segmented images of left and right sclera. These images are then to be normalized to ensure equal dimension for every iteration. In this case, we applied Daugman's rubber sheet normalization method [1]. The normalized images will have the size of $30 \times 240$ pixels. All these steps are illustrated in Figure 3. Next, any presence of eyelash is removed using inpainting algorithm [32]. This is done by replacing the eyelash pixels with the mean of surrounding pixels. We observed that either one of the left or right normalized image will have a slightly brighter or darker lens boundary. This is due to the inconsistent illumination caused by poor flash lighting throughout the enrollment process. In order to overcome this, we proposed a method called summed-histogram, where the frequencies for each pixel's intensity value in the whole image is summed. Whenever the sum value is greater, the normalized image is considered to have brighter lens boundary compared to the other. The summedhistogram of individual normalized image, $S$ is represented as follows:

$$
S_{I(r, \theta)}=\int_{n=0}^{255} \sum P_{I(r, \theta)_{n}}+P_{I(r, \theta)_{n+1}}+P_{I(r, \theta)_{n+\cdots}}
$$

Where $S$ is the summed-histogram of normalized image $I$ and $P_{I}$ is the pixel's intensity in the corresponding normalised polar coordinates $(r, \theta)$.The value of $S$ will be the preliminary input for the next segmentation process. In this process, the ridge detection algorithm is employed to segment the lens boundary on the normalized image. Among others segmentation algorithm, ridge detection has the ability to detect edge while sharing the same background colour but differ in intensities, which resembles the lens boundary [33]. The output from the ridge detection algorithm is the segmented lens boundary in a form of binary image where lens boundary will be regarded as white (1) and the background as black (0). For without soft lens images, a blank image of black coloured will be generated. 


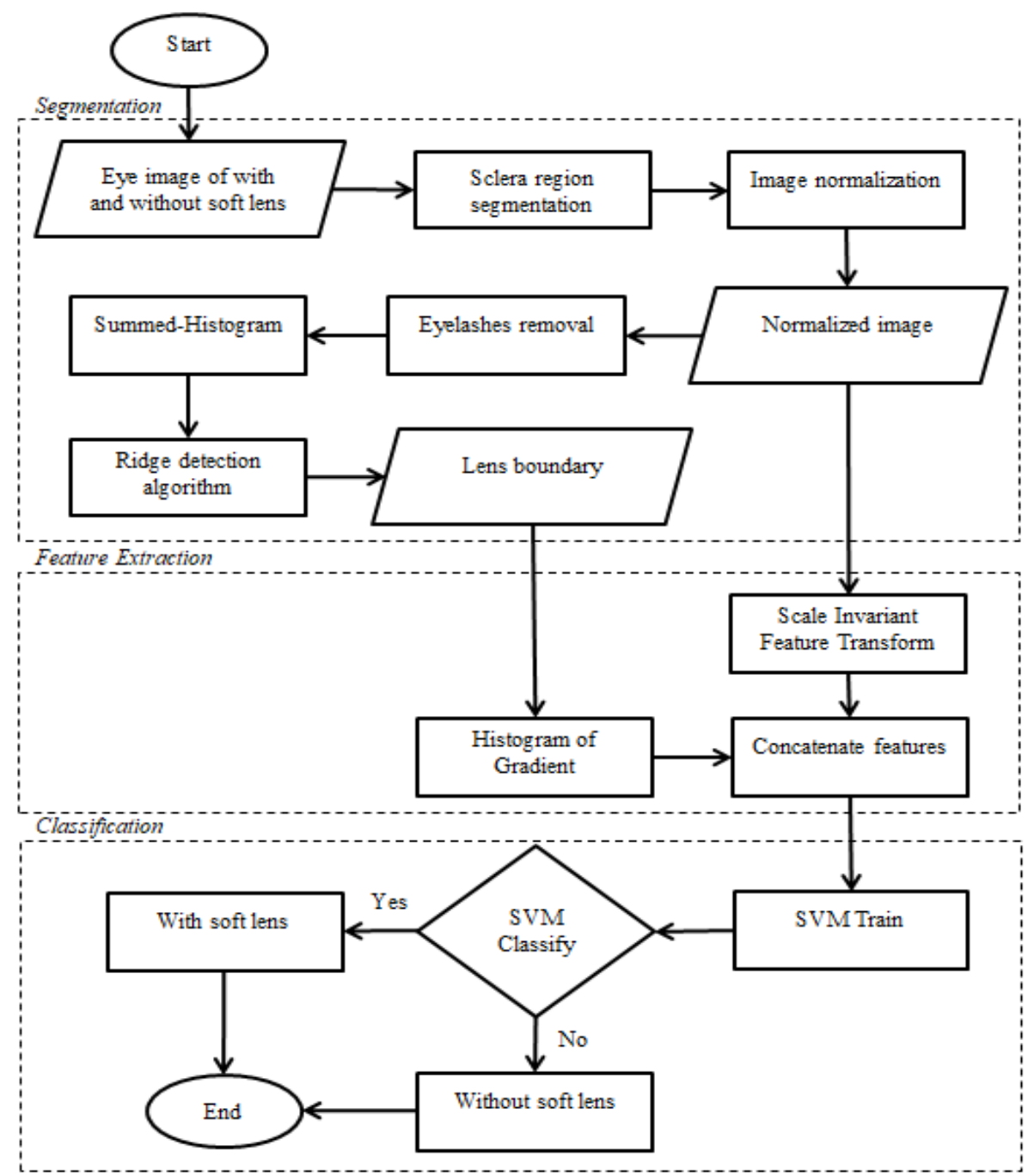

Figure 2. The proposed two-class contact lens classification process

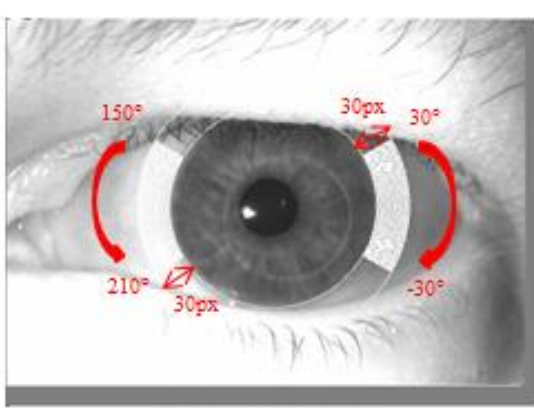

Sclera segmentation

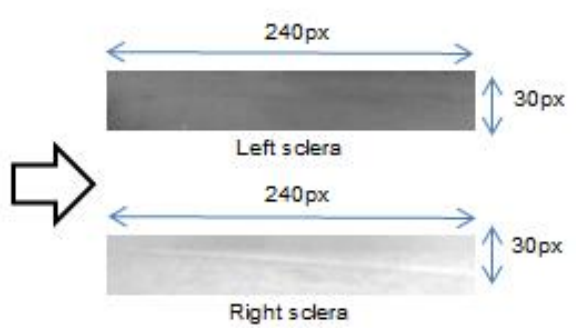

Image normalization

Figure 3. The sclera segmentation and image normalization process

\subsection{Feature Extraction}

Under feature extraction stage, two input images are required; segmented lens boundary image and normalized image of sclera region. The segmented lens boundary image is extracted using a window based feature descriptor; Histogram of Gradient [34]. HOG is chosen as the segmented lens boundary has the properties of the edge orientation. By this mean, for every $n \times n$ pixel in the image, the frequency histogram 
of edge orientation is computed. Then, the resulted edge orientation is quantized into $b$ bins. In our experiment, $n$ and $b$ are set to 4 and 9 respectively. From our observation, the gradient of lens boundary is prone to appear in horizontal scale, which 170 to 190 degree rotation. Meanwhile, the normalized image of the sclera region is descripted using Scale Invariant Feature Transform (SIFT) [35]. The utilization of SIFT is to adapt the properties of having different scales, rotation and inhomogeneous shape of lens boundary, without degrading the performance of detection. There are four main steps of SIFT; scale-space representation, keypoints detection, keypoints orientation and generate keypoint descriptor. During scalespace representation, the Gaussian blurring is applied to the whole image with the number of octave is 4 and level per octave are set to 3.Then, Difference of Gaussian (DoG) of the blurred image is obtained by subtracting subsequent scales in each octave, producing multiple image point of $D(x, y, \sigma)$. By this mean, a keypoint is detected when its value is smaller (local minimum) or larger (local maximum) than the surrounding point. Poorly localized points are excluded during keypoints detection. The next process is to assign an orientation to each keypoint by calculating its gradient directions and magnitudes in a region of 16 $\times 16$. Finally, these regions are broken into sixteen $4 \times 4$ window and accumulates them into 8 bins histogram with a weighted value of gradient magnitude. Therefore, there is $4 \times 4 \times 8=128$ feature vectors for each keypoint. In this work, our interest is to use the description of edge orientation of HOG and the invariance edge properties through SIFT to form a distinguishable feature of without and with soft lens. This is done by concatenating both left and right features of HOG and SIFT for the respective iris image into a feature vector.

\subsection{Classification}

The concatenated features are trained using a non-linear SVM with radius basis function kernel. 10 fold cross validation were utilized to obtain the fittest parameters of $C$ and gamma in order to reduce overfitting. In order to classify between without or with soft lens, the images of without lens are also applied to the whole process from segmentation to feature extraction. Images with prior knowledge of soft lens presence will be labelled as positive samples and without lens images as negative samples.

\section{RESULTS AND ANALYSIS}

In this work, the iris images are retrieved from Notre Dame Cosmetic Contact Lens 2013 (NDCCL13) [31] that contains grayscale iris images of without lens, with soft lens and with cosmetic lens. All images are captured using either LG4000 or AD100 NIR camera. For LG4000, all images are split into 3000 for training and 1200 for testing while for AD100, 600 images are for training and 300 for testing. Table 1 shows the images distribution for NDCCL13 database.

Table 1. NDCCL13 images class distribution

\begin{tabular}{ccccc}
\hline Camera & Class & Label & Training & Testing \\
\hline \multirow{4}{*}{ LG4000 } & No & N & 1000 & 400 \\
& Soft & S & 1000 & 400 \\
& Cosmetic & T & 1000 & 400 \\
& No & N & 200 & 100 \\
AD100 & Soft & S & 200 & 100 \\
& Cosmetic & T & 200 & 100 \\
\hline
\end{tabular}

All experiments are executed using Matlab R2017b on a machine with $2.3 \mathrm{GHz}$ and $6 \mathrm{~GB}$ memory. We calculated confusion matrix for each class of different cameras. For uniformity with [7], we only reported correct classification rate (CCR) with its average. However, the evaluation of cosmetic lens in not within our scope as this work only focusing on two class classification of with or without soft lens. We annotated the class of without lens as $\mathrm{N}$ and soft lens as $\mathrm{S}$. N-N refers to the probability of without lens samples are classified belongs to without lens while S-S refers to the probability of soft lens samples are classified belongs to soft lens. Comparisons are made with existing methods proposed in [7] by using classical LBP, [36] by using a modified version of LBP, [28] by using Convolutional Neural Network, [27] by using Binarized Statistical Image Features and [25] by using Scale Invariant Descriptor. During segmentation stage, each iris image will produce two segmented lens boundary images and two normalized images which derived from the left and right sclera. These images are used for training and testing throughout the classification stage. These samples of images are shown in Table 2. Therefore, for LG4000, there are 2000 training images for without lens and another 2000 for soft lens. Meanwhile, for AD100, both without lens and soft lens constitute 400 training images each. The numbers of testing images are doubled up, the same manner as training images. 
Table 2. NDCCL13 images class distribution

\begin{tabular}{|c|c|c|c|}
\hline Image & Sclera & Normalized image & Segmented lens boundary \\
\hline \multirow{2}{*}{$04261 \mathrm{~d} 1016$} & Left & & \\
\hline & Right & & \\
\hline \multirow{2}{*}{$06008 \mathrm{~d} 58$} & Left & & \\
\hline & Right & & \\
\hline
\end{tabular}

Table 3 reports the performance of the proposed method with the aforementioned state-of-the-art methods. The best results are written in bold. In summary, the proposed method has yielded the highest average for both cameras. However, the LG4000's CCR for N-N classification has unable to surpass the method from [25]. In the meantime, the CCR for S-S classification resulted over 10 points from the nearest figure. It is observed that the results for AD100 are very marginal to achieve significant performance. We believe the less number of subjects is not sufficient to statistically generalize the variability between classes. Some of future works $[37,38]$ may be added for better performance in this research.

Table 3. The correct classification rate and average of the proposed and state of the art methods

\begin{tabular}{ccccccccc}
\hline \multirow{2}{*}{ Cameras } & Classification & LBP [7] & $\begin{array}{c}\text { LBP+PHOG } \\
{[7]}\end{array}$ & $\begin{array}{c}\text { mLBP [7] } \\
{[36]}\end{array}$ & CNN [28] & $\begin{array}{c}\text { BSIF [27] } \\
\text { [25] }\end{array}$ & $\begin{array}{c}\text { PID } \\
\text { Method }\end{array}$ \\
\hline \multirow{3}{*}{ LG4000 } & N-N & 70.00 & 81.25 & 85.50 & 84.50 & 76.50 & 95.75 \\
& S-S & 60.15 & 65.41 & 45.25 & 73.75 & 84.50 & 84.00 \\
& Average & 65.08 & 73.33 & 65.38 & 79.13 & 80.50 & 89.88 & 95.75 \\
AD100 & N-N & 42.00 & 42.00 & 81.00 & 73.00 & 79.50 & 79.00 & 80.20 \\
& S-S & 54.00 & 60.00 & 52.00 & 65.00 & 62.00 & 78.00 & 79.12 \\
& Average & 48 & 51 & 66.5 & 69 & 70.75 & 78.5 & $\mathbf{7 9 . 6 6}$ \\
\hline
\end{tabular}

\section{CONCLUSION}

This work proposed two class contact lens classification of with or without soft lens. In order to distinguish between these classes, we focused on extracting local information available on the sclera region. It is observed that the wearing of soft lens may leave thin lines which appear to be the lens boundary. Therefore, we proposed a segmentation mechanism to segment this boundary and utilize Histogram of Gradient to extract the gradient orientation of the boundary. We also descript the original sclera region with Scale Invariant Feature Transform in order to tackle the inhomogeneous shape and rotation of the lens boundary. These features are concatenated before trained and classified using a non-linear SVM. Results showed that the proposed method achieved the highest average accuracy compared to other state-of-the-art methods.

\section{ACKNOWLEDGEMENT}

This work has been funded by Universiti Teknologi Malaysia and Ministry of Higher Education Malaysia under FRGS Vot No: 4F973.

\section{REFERENCES}

[1] Daugman JG. Biometric Personal Identification System based on Iris Analysis. Google Patents. 1994.

[2] Dhavale SV. Robust Iris Recognition based on Statistical Properties of Walsh Hadamard Transform Domain. IJCSI International Journal of Computer Science Issues. 2012; 9(2).

[3] Hosseini MS, Araabi BN, and Soltanian-Zadeh H. Pigment Melanin: Pattern for Iris Recognition, IEEE Transactions on Instrumentation and Measurement. 2010; 59(4): 792-804.

[4] Sim HM, Hishammuddinn A, Hassan R, Othman RM. Multimodal Biometrics: Weighted score level fusion based on non-ideal iris and face images. Expert Systems with Applications. 2014; 41(11): 5390-5404.

[5] Wang Y, Han J. Iris Recognition using Support Vector Machines. International Symposium on Neural Networks. 2004; 3173; 622-628.

[6] Baker SE, Hentz A, Bowyer KW, Flynn PJ. Degradation of Iris Recognition Performance due to Non-Cosmetic Prescription Contact Lenses. Computer Vision and Image Understanding. 2010; 114(9): 1030-1044. 
[7] Yadav D, Kohli N, Doyle JS, Singh R, Vatsa M, Bowyer KW. Unraveling the Effect of Textured Contact Lenses on Iris Recognition. IEEE Transactions on Information Forensics and Security. 2014; 9(5): 851-862.

[8] Kalsoom S, Ziauddin S. Iris Recognition: Existing Methods and Open Issues. The Fourth International Conferences on Pervasive Patterns and Applications. 2012: 23-28.

[9] Doyle JS, Flynn PJ, Bowyer KW. Automated Classification of Contact Lens Type in Iris Images, 2013 International Conference on Biometrics (ICB). 2013.

[10] Lovish, Nigam A, Kumar B, Gupta P. Robust Contact Lens Detection using Local Phase Quantization and Binary Gabor Pattern. 16th International Conference Computer Analysis of Images and Patterns. 2015: 702-714.

[11] Kumar B, Nigam A, Gupta P. Fully Automated Soft Contact Lens Detection from NIR Iris Images. International Conference on Pattern Recognition Applications and Methods. 2016: 589-596.

[12] Daugman J. Demodulation by Complex-Valued Wavelets for Stochastic Pattern Recognition. International Journal of Wavelets, Multiresolution and Information Processing. 2003: 1(01); 1-17.

[13] Lee EC, Park KR, Kim J. Fake Iris Detection by Using Purkinje Image. International Conference on Biometrics. 2006: 397-403.

[14] He X, An S, Shi P. Statistical Texture Analysis-based Approach for Fake Iris Detection using Support Vector Machines. International Conference on Biometrics. 2007: 540-546.

[15] Wei Z, Qiu X, Sun Z, Tan T. Counterfeit Iris Detection based on Texture Analysis. 19th International Conference on Pattern Recognition. 2008.

[16] Zhang H, Sun Z, Tan T. Contact Lens Detection based on Weighted LBP. 20th International Conference on. Pattern Recognition (ICPR). 2010.

[17] Williams GO. Iris Recognition Technology. 30th Annual International Carnahan Conference. 1996.

[18] Negin M, Chmielewski TA, Salganicoff M, von Seelen UM, Venetainer PL, Zhang GG. An Iris Biometric System for Public and Personal Use. Computer. 2000: 33(2); 70-75.

[19] Ali JM, Hassanien AE. An Iris Recognition System to Enhance e-Security Environment based on Wavelet Theory. Advanced Modeling and Optimization. 2003: 5(2); 93-104.

[20] Baker SE, Hentz A, Bowyer KW, Flynn PJ. Contact Lenses: Handle with Care for Iris Recognition. 2009 IEEE 3rd International Conference on Biometrics: Theory, Applications and Systems. 2009: 190-197.

[21] Kywe W, Yoshida M, Murakami K. Contact Lens Extraction by Using Thermo-Vision. 18th International Conference on Pattern Recognition. 2006.

[22] Lee EC, Park KR, Kim J. Fake Iris Detection by using Purkinje Image. Advances in Biometrics. 2005: 397-403.

[23] Hughes K, Bowyer KW. Detection of Contact Lens-based Iris Biometric Spoofs using Stereo Imaging. 46th Hawaii International Conference on System Sciences (HICSS). 2013.

[24] Kohli N, Daksha Y, Vatsa M, Singh R. Revisiting Iris Recognition with Color Cosmetic Contact Lenses. International Conference on Biometrics. 2013: 1-5.

[25] Gragnaniello D, Poggi G, Sansone C, Verdoliva L. Contact Lens Detection and Classification in Iris Images through Scale Invariant Descriptor. 10th International Conference on Signal-Image Technology and InternetBased Systems. 2014: 560-565.

[26] Gragnaniello D, Poggi G, Sansone C, Verdoliva L. Using Iris and Sclera for Detection and Classification of Contact Lenses. Pattern Recognition Letters. 2016: 82(2); 251-257.

[27] Raghavendra R, Raja KB, Busch C. Ensemble of Statistically Independent Filters for Robust Contact Lens Detection in Iris Images. Indian Conference on Computer Vision Graphics and Image Processing. 2014.

[28] Silva P, Luz E, Baeta R, Pedrini H, Falcao AX, Menotti D. An Approach to Iris Contact Lens Detection based on Deep Image Representations. 28th SIBGRAPI Conference on Graphics, Patterns and Images. 2015: 157-164.

[29] Erdogan G, Ross A. Automatic Detection of Non-Cosmetic Soft Contact Lenses in Ocular Images. Biometric and Surveillance Technology for Human and Activity Identification X, 2013: 8712.

[30] He Z, Sun Z, Tan T, Wei Z. Efficient Iris Spoof Detection via Boosted Local Binary Patterns. International Conference on Biometrics. 2009: 1080-1090.

[31] Doyle J, Bowyer K. Notre Dame Image Database for Contact Lens Detection In Iris Recognition-2013. 2014.

[32] Bertalmio M, Sapiro G, Caselles V, Ballester C. Image Inpainting. 27th annual conference on Computer Graphics and Interactive Techniques. 2000: 417-424.

[33] Damon J. Properties of Ridges and Cores for Two-Dimensional Images, Journal of Mathematical Imaging and Vision. 1999: 10(2); 163-174.

[34] Dalal N, Triggs B. Histograms of Oriented Gradients for Human Detection. IEEE Computer Society Conference on Computer Vision and Pattern Recognition. 2005: 1; 886-893.

[35] Lowe DG. Distinctive Image Features from Scale-Invariant Keypoints. International Journal of Computer Vision. 2004: 60(2); 91-110.

[36] Doyle JS, Bowyer KW, Flynn PJ. Variation in Accuracy of Textured Contact Lens Detection based on Sensor and Lens Pattern. IEEE Sixth International Conference on Biometrics: Theory, Applications and Systems. 2013: 1-7.

[37] Gunawan, T. S., Solihin, N. S., Morshidi, M. A., \& Kartiwi, M. (2017). Development of Efficient Iris Identification Algorithm using Wavelet Packets for Smartphone Application. Indonesian Journal of Electrical Engineering and Computer Science, 8(2).

[38] Chen, D., \& Qin, G. (2017). An Embedded Iris Image Acquisition Research. Indonesian Journal of Electrical Engineering and Computer Science, 5(1), 90-98. 